\title{
FutureJournal
}

\section{Análise de Cenários Envolvendo Satisfação no Trabalho e Estresse Ocupacional por meio da Dinâmica de Sistemas}

\author{
Damiana Machado de Almeida \\ Doutoranda do Programa de Pós-Graduação em Administração (PPGA) da Universidade \\ Federal de Santa Maria (UFSM) \\ dimyalmeida@gmail.com \\ Gean Carlos Tomazzoni \\ Doutorando do Programa de Pós-Graduação em Administração da Universidade Federal do \\ Rio Grande do Sul (UFRGS) \\ gean.tomazzoni@hotmail.com
}

\section{Andressa Schaurich dos Santos}

Doutoranda do Programa de Pós-Graduação em Administração (PPGA) da Universidade

Federal de Santa Maria (UFSM) dessaschaurich@gmail.com

\section{Glauco Oliveira Rodrigues}

Doutoranda do Programa de Pós-Graduação em Administração (PPGA) da Universidade Federal de Santa Maria (UFSM) glaucop10@redes.ufsm.br

\section{Eugênio de Oliveira Simonetto}

Professor adjunto no Departamento de Ciências Administrativas da Universidade Federal de Santa Maria (UFSM) eosimonetto@gmail.com

\section{RESUMO}

Este artigo tem por objetivo apresentar o desenvolvimento e a validação de um modelo de simulação computacional que permita aos gestores da área de gestão de pessoas avaliarem e analisarem cenários acerca da satisfação no trabalho e do estresse ocupacional. A partir das relações inversamente proporcionais entre estresse e satisfação, estabeleceu-se uma associação entre as duas variáveis, considerando-se um impacto positivo da satisfação para a redução dos níveis de estresse. Diante da análise dessas relações, percebeuse que, ao longo dos 10 períodos simulados, o aumento da satisfação em $14,8 \%$ gerou uma redução nos níveis de estresse ocupacional. Assim, o estresse foi reduzido de uma média de 2,16 a um nível de 2,05 . Os resultados desta pesquisa proporcionam uma importante compreensão acerca das relações da satisfação no trabalho com estresse ocupacional e fornecem um modelo de simulação que testa os impactos das ações desenvolvidas pela área 


\section{FutureJournal}

de gestão de pessoas para elevar os níveis de satisfação sobre os níveis de estresse ocupacional de seus colaboradores.

PALAVRAS-CHAVE: Estresse ocupacional. Satisfação no trabalho.

Dinâmica de sistemas.

\section{Scenario Analysis Involving Satisfaction in Work and Occupational Stress through System Dynamics}

\section{ABSTRACT}

This article presents the development and validation of a computer simulation model that enables people management area managers to assess and analyze scenarios about job satisfaction and occupational stress. From the inversely proportional relationship between stress and satisfaction, we established an association between the two variables, considering the positive impact of satisfaction for reducing stress levels. After analyzing these relationships, it was observed that the increased satisfaction by $14.8 \%$ over the 10 simulated periods generated a reduction in the levels of occupational stress. Thus, the stress was reduced from an average of 2.16 at a level of 2.05. The results of this research provide important insight about the satisfaction relationships at work with occupational stress, providing a simulation model to test the impact of actions developed by the people management area to raise levels of satisfaction about occupational stress levels its employees.

KEY-WORDS: Occupational stress. Job satisfaction. System dynamics. 


\section{INTRODUÇÃO}

A busca incessante por uma melhor qualidade de vida como forma de satisfação profissional ou pessoal tem sido alvo principal do ser humano. As tecnologias, o ritmo de vida acelerado, a pressão por resultados e a estrutura de trabalho são alguns dos aspectos que podem ser citados como os que atingem diretamente os indivíduos na atualidade. O estresse, intimamente ligado a esses fatores, tem se tornado um problema nas organizações, com o aumento das exigências dos cargos, impactando na satisfação do colaborador frente à atividade exercida ou ao setor em que se trabalha.

Devido à sobrecarga de um mercado de trabalho cada vez mais exigente, os indivíduos dentro das organizações estão cada vez mais críticos no que se refere às tarefas que estão desempenhando e à sua eficiência. Consequentemente, o estresse ligado a esse fator tem se tornado um problema mais frequente nas organizações e na vida dos funcionários. De acordo com Paschoal e Tamayo (2004), o estudo sobre o estresse no trabalho tem crescido na literatura científica nos últimos anos devido ao seu impacto negativo sobre a saúde e o bem-estar do trabalhador, e sobre a produtividade e o funcionamento da organização. O Ministério da Saúde e a Organização Pan-Americana da Saúde no Brasil (Brasil, 2001) ressaltam que esse é um tema que toma espaço nesse cenário, exigindo cada vez mais pesquisas para que se o conheça, com mais profundidade e para que se possam traçar estratégias efetivas de prevenção.

Diante desse contexto, outra temática que merece atenção é a satisfação no trabalho, que, para Siqueira (2008), se refere ao vínculo afetivo do indivíduo com o seu labor, havendo evidências de que a satisfação no trabalho está ligada a fatores sócio-organizacionais como valores, percepções de justiça, de suporte e de reciprocidade que se estabelecem a partir das trocas entre indivíduo, colegas e organização. Sendo assim, a satisfação no trabalho está diretamente relacionada às políticas e práticas gerenciais da organização, das quais depende.

Em face dessas considerações, emerge como objetivo desta investigação apresentar o desenvolvimento e a validação de um modelo de 
simulação computacional que permita aos gestores da área de gestão de pessoas avaliarem e analisarem cenários acerca da satisfação no trabalho e do estresse ocupacional.

Conforme destacam Paschoal e Tamayo (2004), o trabalho integrase como algo de grande importância à vida de qualquer pessoa, pois está intimamente relacionado à função de suprir as necessidades básicas do ser humano. Além disso, o tempo que um indivíduo dedica ao seu trabalho, ocupa a maior parte de sua vida. Devido a esse fato, a preocupação com o bem-estar no trabalho e, consequentemente, a satisfação com ele, deve ser algo discutido e priorizado como fundamental para que o indivíduo consiga desenvolver sua função dentro a organização.

No cenário brasileiro, é notória a falta de atenção em relação à saúde dos trabalhadores do setor de serviços, e no caso dos policiais militares não é diferente. Para Costa, Accioly, Oliveira e Maia (2007), dentre os profissionais que estão mais propensos ao estresse no trabalho, em função da pressão e dos riscos das atividades diárias, estão os policiais militares, uma vez que esses profissionais trabalham sob forte tensão em situações que colocam em risco a sua vida. Contudo, apesar da importância do estudo desse tema na classe de policiais militares, tanto para o indivíduo, quanto para o governo estadual e para a sociedade em geral, é comprovado que esses profissionais não vêm sendo pesquisados em profundidade.

Na visão de Vasconcelos (2011), faz-se necessária a realização de pesquisas com esses profissionais a fim de que os gestores possam conhecer e compreender as respostas do seu efetivo em relação ao estresse, a traumas e ao abuso de substâncias. Só assim será possível intervir, implementando as estratégias e os tratamentos necessários para auxiliar esses policiais militares a sobreviverem ao rigor do contexto ocupacional.

Ademais, no que se refere à satisfação no trabalho, saber o que satisfaz o colaborador é importante, também, principalmente para auxiliar na definição de procedimentos a serem adotados que busquem aprimorar o desempenho, tanto individual quanto da instituição. Assim, espera-se que, com os resultados encontrados no presente estudo, seja possível traçar 
estratégias que busquem reduzir o nível de estresse, bem como evitar que indivíduos venham a desenvolvê-lo, e melhorar a satisfação e, conjuntamente, a qualidade de vida dos indivíduos dentro das organizações.

Para tanto, o presente artigo está organizado em mais cinco seções, além desta introdução e das referências, que são apresentadas ao final do estudo. Nas seções 2 e 3, são apresentados o método de pesquisa utilizado para o desenvolvimento do estudo, bem como o problema de modelagem, as variáveis componentes e o modelo desenvolvido. $\mathrm{Na}$ seção 4, são apresentados a validação, os cenários de simulação e o experimento utilizando o modelo; na seção 5, são apresentados os resultados gerados pelo modelo para o experimento. Por fim, na seção 6, são destacadas as conclusões acerca da pesquisa, trazendo-se os desfechos do estudo e sintetizando-se seus principais resultados e contribuições, bem como suas limitações e implicações para investigações futuras.

\section{MÉTODO DA PESQUISA}

A metodologia de pesquisa adotada para o desenvolvimento do modelo computacional foi baseada em Law e Kelton (1991) e consistiu em cinco etapas: (1) levantamento exploratório em artigos científicos, dissertações e livros, com os quais foi caracterizado o problema, e objetivo de pesquisa; (2) desenvolvimento da solução, pela construção de modelos formais capazes de representar o problema; (3) implementação computacional da solução, utilizando-se o software Vensim (VensimVentana, 2012) da área de system dynamics; (4) validação da solução, por meio de análise de especialistas da área de gestão de pessoas, para verificar se os resultados obtidos estão de acordo com a realidade observada, bem como por meio da (5) simulação de um experimento utilizando-se dois cenários.

Os cenários utilizados para a validação do modelo e a formulação da hipótese dinâmica e os conceitos envolvidos no desenvolvimento da pesquisa são apresentados nas subseções 2.1 e 2.1.1.

\subsection{SATISFAÇÃO NO TRABALHO E ESTRESSE OCUPACIONAL}


O "cenário atual" do presente estudo foi baseado no estudo de Almeida (2015), realizado com os policiais militares que fazem parte da Brigada Militar no Estado do Rio Grande do Sul. A população do estudo foi composta por 33.650 servidores militares, e a amostra constituída por 519 indivíduos que trabalham em 97 municípios do estado do Rio Grande do Sul.

O instrumento utilizado para avaliar o estresse ocupacional e a satisfação no trabalho foi dividido em duas partes: Parte I - Escala de Estresse no Trabalho (EET), composta por 23 itens e validada por Paschoal e Tamayo (2004); e Parte II - Escala de Satisfação no Trabalho (EST), composta por 25 itens e validada por Siqueira (2008), conforme apresentado na Figura 1.

A EET foi elaborada a partir da análise de literatura sobre estressores organizacionais de natureza psicossocial e sobre reações psicológicas ao estresse ocupacional. Cada item aborda tanto um estressor quanto uma reação a ele. Já a EST analisa o contentamento no trabalho a partir de cinco dimensões constituídas cada uma por cinco itens: satisfação com os colegas de trabalho (Fator 1), satisfação com o salário (Fator 2), satisfação com a chefia (Fator 3 ), satisfação com a natureza do trabalho (Fator 4 ) e satisfação com as promoções (Fator 5).

Importante ressaltar que, para responder aos questionamentos da EET de Paschoal e Tamayo (2004), fez-se uso da escala do tipo Likert de cinco pontos, variando de 1 (discordo totalmente) a 5 (concordo totalmente). Já para a EST de Siqueira (2008), utilizou-se a escala do tipo Likert de sete pontos, variando de 1 (totalmente insatisfeito) a 7 (totalmente satisfeito). 


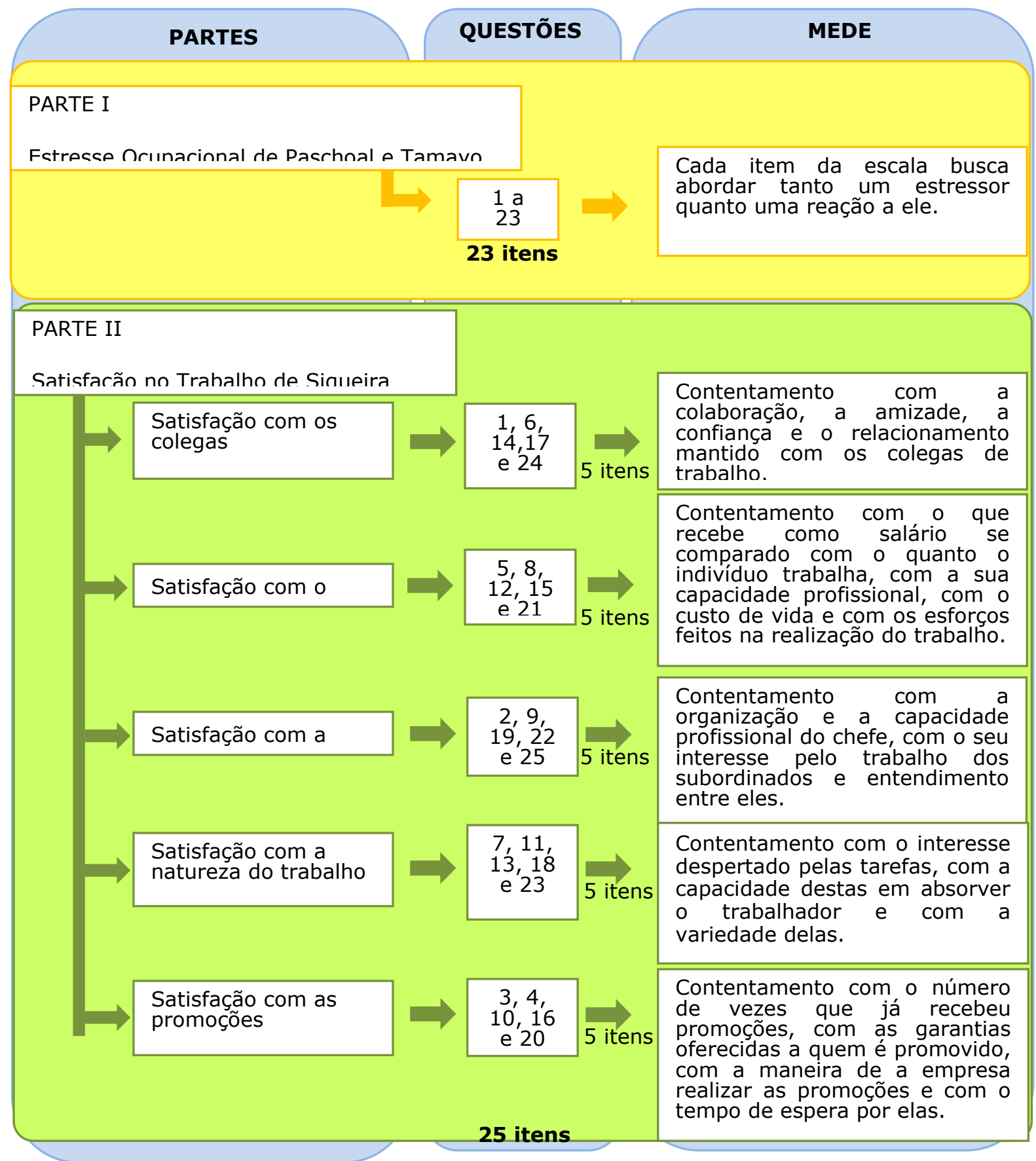

Figura 1: Representação do questionário

Fonte: Os autores

\subsubsection{Dinâmica de sistemas (system dynamics)}

De acordo com Daellenbach e McNickle (2005), a metodologia system dynamics (SD) permite o estudo do comportamento dos sistemas ao longo do tempo, de maneira a permitir a avaliação das consequências das decisões. Diante disso e pela intenção de se investigarem cenários de 
satisfação no trabalho e estresse ocupacional em um horizonte temporal futuro, decidiu-se utilizá-la na modelagem e simulação computacional.

\section{O MODELO DE SIMULAÇÃO}

Os achados de Almeida (2015) serviram como base para este estudo, no qual se identificou que o estresse ocupacional apresenta correlação negativa e estatisticamente significante com a satisfação no trabalho e suas cinco dimensões. Ressalta-se que, conforme este estudo, as dimensões da satisfação que apresentaram menores médias foram satisfação com o salário e satisfação com as promoções, sendo estas as que merecem atenção por parte da Brigada Militar.

Diante disso, no presente trabalho, buscou-se o desenvolvimento de um modelo de simulação que permitisse aos gestores da área de gestão de pessoas avaliar políticas e práticas que possam contribuir para a melhoria do "cenário atual". Assim, buscou-se manipular as variáveis "satisfação com as promoções" e "satisfação com o salário", dado que esses fatores apresentaram as menores médias, e, a partir daí, avaliar o impacto nos níveis de estresse ocupacional.

Na Figura 2, apresenta-se a construção do modelo de simulação desenvolvido para este estudo. Evidencia-se que esse modelo é composto por duas variáveis principais - satisfação no trabalho e estresse ocupacional -, além de quatro variáveis auxiliares para o estresse e cinco para a satisfação, representando construtos de cada uma das variáveis principais. 


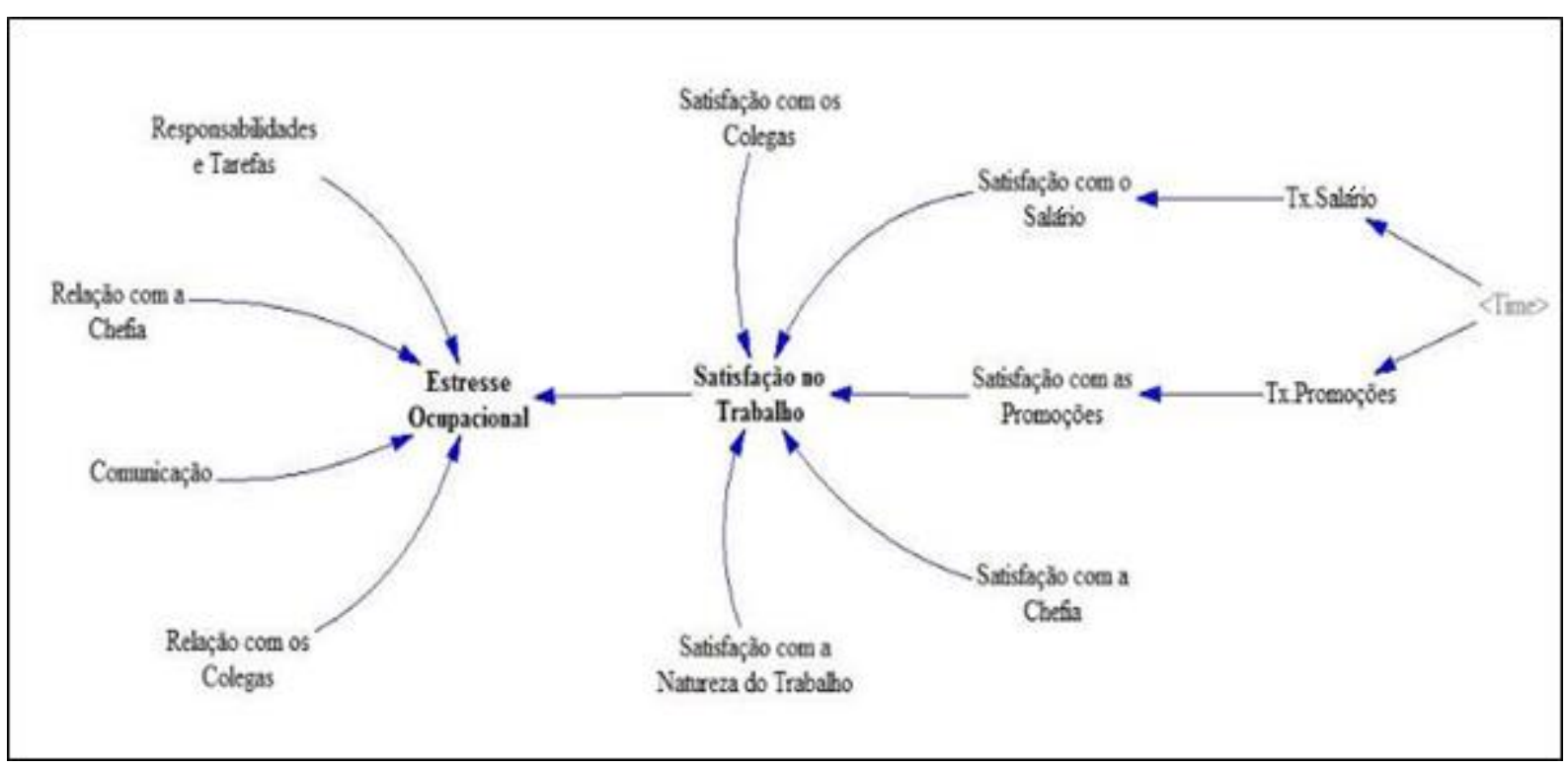

Figura 2: Modelo de simulação desenvolvido

Fonte: Elaborado pelos autores

Acredita-se que, por meio das análises geradas pelo modelo, as decisões poderão envolver a busca pela elevação da taxa salário (satisfação com o salário) e da taxa promoções (satisfação com as promoções).

\section{VALIDAÇÃO E EXPERIMENTOS DO MODELO DE SIMULAÇÃo}

Na validação conceitual do problema, primeira fase, foram validados os requisitos e as variáveis do modelo. Para tanto, utilizaram-se dados de artigos científicos, dissertações e livros, além de contar-se com a participação e a avaliação de especialistas da área de gestão de pessoas. Na segunda fase de validação, que se refere à implantação no simulador Vensim (Vensim-Ventana, 2012), foram utilizadas as escalas validadas por Paschoal e Tamayo (2004) e Siqueira (2008), acerca do estresse ocupacional e da satisfação no trabalho, respectivamente, por meio do estudo de Almeida (2015).

Além disso, também foram verificados e validados os resultados gerados pelo modelo de simulação e sua conformidade com resultados obtidos no mundo real, denotando a fidedignidade do modelo. Em ambas as fases, os resultados atenderam às expectativas dos pesquisadores da simulação e de especialistas. 
Na etapa de validação posterior, para a construção do experimento, foram utilizadas taxas reais (médias das dimensões satisfação com o trabalho) na perspectiva dos policiais militares do estado do Rio Grande do Sul. Para tal, foram gerados dois cenários simulados no modelo - (a) "cenário atual" e (b) "cenário otimista" -, o qual é baseado em taxas crescentes da satisfação com o salário, bem como da satisfação com as promoções. As demais médias permaneceram estáticas.

Esse tipo de validação pode ser reconhecido como análise de sensibilidade do modelo de simulação, visto que foram utilizadas variáveis controladas nos inputs e verificadas as variações nos resultados. $O$ detalhamento e a quantificação de taxas para cada cenário simulado são apresentados na subseção 4.1 .

\subsection{CENÁRIOS SIMULADOS NO MODELO}

Buscando melhor visualização dos cenários simulados, a Tabela 1 apresenta as médias referentes às dimensões da satisfação no trabalho. São elas: satisfação com os colegas, com o salário, com a chefia, com a natureza do trabalho e com as promoções.

Tabela 1: Estatística descritiva da satisfação no trabalho e das respectivas dimensões

\begin{tabular}{lcc}
\hline \multicolumn{1}{c}{ Modelos Teóricos } & Cenário Atual & $\begin{array}{c}\text { Cenário } \\
\text { Otimista }\end{array}$ \\
\hline Satisfação com os colegas & 5,00 & 5,00 \\
\hline Satisfação com o salário & $\mathbf{2 , 7 9}$ & $\mathbf{4 , 1 8}$ \\
\hline Satisfação com a chefia & 4,42 & 4,42 \\
\hline Satisfação com a natureza do trabalho & 4,37 & 4,37 \\
\hline Satisfação com as promoções & $\mathbf{3 , 0 3}$ & $\mathbf{4 , 5 4}$ \\
\hline
\end{tabular}

Fonte: Dados da pesquisa

Para o "cenário otimista", considerou-se um crescimento de $50 \%$ do "cenário atual" nas médias referentes à satisfação com o salário e à satisfação com as promoções; as demais médias, conforme evidenciado anteriormente, permaneceram estáticas. Destaca-se que esse crescimento foi gradativo num período de 10 anos. 
$\mathrm{Na}$ Tabela 2, apresenta-se a estatística descritiva do estresse ocupacional e das dimensões. Faz-se importante ressaltar que a escala original EET não apresenta dimensões, assim, as consideradas no presente estudo foram elaboradas especialmente para esse fim, com base no conhecimento dos pesquisadores.

Tabela 2: Estatística descritiva do estresse ocupacional e respectivas dimensões

Modelos Teóricos

Responsabilidade/Tarefas

Relação com a Chefia

Comunicação

Relação com os Colegas
Cenário Atual

3,90

3,61

3,54

3,69

Fonte: Dados da pesquisa

Assim, a partir desse resultado, para mensurar os níveis de estresse, manipulando as variáveis de satisfação no trabalho, desenvolveuse a seguinte equação:

$$
\left.\mathrm{En}=\mathrm{En} \mathrm{n}_{0}-\left((0,014 * \mathrm{n}) * S \mathrm{n}_{0}\right) * 0,20\right)
$$

em que:

$\mathrm{n}$ : tempo em ano;

En: nível de estresse;

$E n_{0}$ : nível de estresse no cenário atual = (média aritmética entre quatro construtos de estresse e a média de satisfação - impacto negativo no cenário atual);

$\mathrm{Sn}_{0}$ : nível de satisfação no cenário atual = (média aritmética dos cinco construtos de satisfação no cenário atual).

\subsection{EXPERIMENTO}

Definidos os cenários para a realização do experimento com o uso do modelo, foram executadas as simulações. Conforme descrito anteriormente, os dados utilizados em ambos os cenários são sob a perspectiva dos policiais militares do estado do Rio Grande do Sul. As demais informações foram obtidas em documentos técnicos.

A simulação considerou um período de tempo de dez anos, o qual pode ser alterado pelos usuários finais ou pelos pesquisadores do modelo, visando a projeções de um período mais curto ou mais longo. Para a 
execução dos cenários simulados, foi utilizado o simulador Vensim (VensimVentana, 2012). Os resultados obtidos com o uso do modelo são apresentados na seção seguinte.

\section{RESULTADOS OBTIDOS}

Nesta seção, serão apresentados os resultados da modelagem referentes à satisfação no trabalho e ao estresse ocupacional, ou seja, o quanto o estresse é impactado pela satisfação no trabalho, em especial, por meio da manipulação das dimensões salário e promoções.

Primeiramente, apresenta-se a análise referente aos níveis de satisfação. Para tal, foram simuladas no modelo ações, em uma perspectiva otimista, voltadas para os fatores "satisfação com as promoções" e "satisfação com os salários" em 50\%, ao longo de dez anos. Os resultados gerados podem ser visualizados na Tabela 3 .

Tabela 3: Níveis de satisfação

\begin{tabular}{c|c|c|c|c|c|c|c|c|c}
\hline \multicolumn{1}{|c}{ Períodos/Anos } \\
\hline $\mathbf{0}$ & $\mathbf{1}$ & $\mathbf{2}$ & $\mathbf{3}$ & $\mathbf{4}$ & $\mathbf{5}$ & $\mathbf{6}$ & $\mathbf{7}$ & $\mathbf{8}$ & $\mathbf{9}$ \\
\hline 3,92 & 3,99 & 4,05 & 4,11 & 4,18 & 4,24 & 4,31 & 4,37 & 4,44 & 4,50 \\
\hline
\end{tabular}

Fonte: Dados da pesquisa

A partir da Tabela 3, verifica-se um aumento considerável nos níveis de satisfação no trabalho, representando, em uma escala de 1 a 7, um aumento de 3,92 para 4,50. Seguindo essa mesma análise, no Gráfico 1, apresenta-se a esquematização gráfica dos resultados contidos na Tabela 3. 


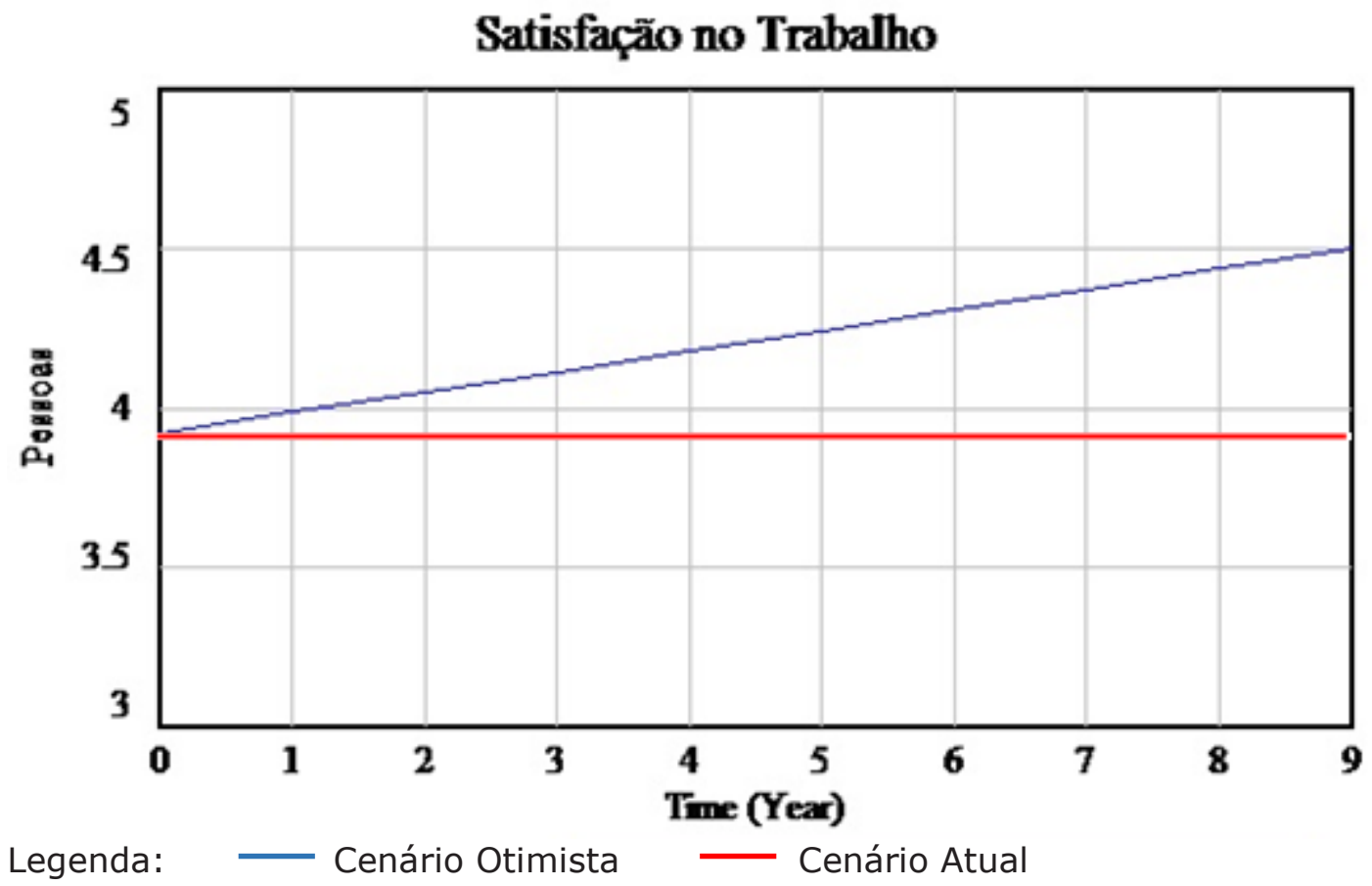

\section{Gráfico 1: Níveis de satisfação no trabalho}

Fonte: Dados da Pesquisa

Com base na análise do Gráfico 1 no modelo de simulação, verificase que, ao se manipularem as variáveis "satisfação com as promoções" e "satisfação com o salário", visando a um aumento de $50 \%$ nos níveis dessas dimensões ao longo de dez períodos, os níveis de satisfação no trabalho crescem, atingindo uma taxa de $14,8 \%$ no final do $10^{\circ}$ período e uma média de 4,5. Esse resultado reforça o estudo realizado por Robbins, Judge e Sobral (2010), que afirmam que a insatisfação com os salários e com as possibilidades de promoção são as questões que mais aparecem nas pesquisas sobre satisfação no trabalho.

Além da tendência de insatisfação com o salário, a tendência de insatisfação com as promoções também foi identificada. Segundo Chanlat (2005), os indivíduos que fazem parte de uma organização merecem explicações a respeito das promoções, além de um processo de promoção equitativo e o mais claro possível. Assim, tais resultados comprovam a necessidade de as organizações desenvolverem ações e práticas voltadas para esses fatores, visando otimizar os níveis gerais de satisfação no trabalho, como demonstrado no Gráfico 1. 
Dado o objetivo deste estudo, que é o de analisar cenários acerca da satisfação no trabalho e do estresse ocupacional, visando identificar a influência da satisfação no trabalho sobre os níveis de estresse ocupacional, apresentam-se, na Tabela 4, os resultados da simulação do impacto gerado a partir do melhoramento dos níveis de satisfação no trabalho, causados pela manipulação, em uma perspectiva otimista, das variáveis "satisfação com as promoções" e "satisfação com o salário", no decorrer dos dez períodos em estudo.

\section{Tabela 4: Níveis de estresse ocupacional}

\begin{tabular}{c|c|c|c|c|c|c|c|c|c}
\hline \multicolumn{1}{|c|}{ Período/Anos } \\
\hline $\mathbf{0}$ & $\mathbf{1}$ & $\mathbf{2}$ & $\mathbf{3}$ & $\mathbf{4}$ & $\mathbf{5}$ & $\mathbf{6}$ & $\mathbf{7}$ & $\mathbf{8}$ & $\mathbf{9}$ \\
\hline 2,16 & 2,15 & 2,14 & 2,13 & 2,11 & 2,10 & 2,09 & 2,07 & 2,06 & 2,05 \\
\hline
\end{tabular}

Já no Gráfico 2, apresenta-se a representação dos níveis de estresse ocupacional gerados no cenário simulado.

\section{Estresse Ocupacional}

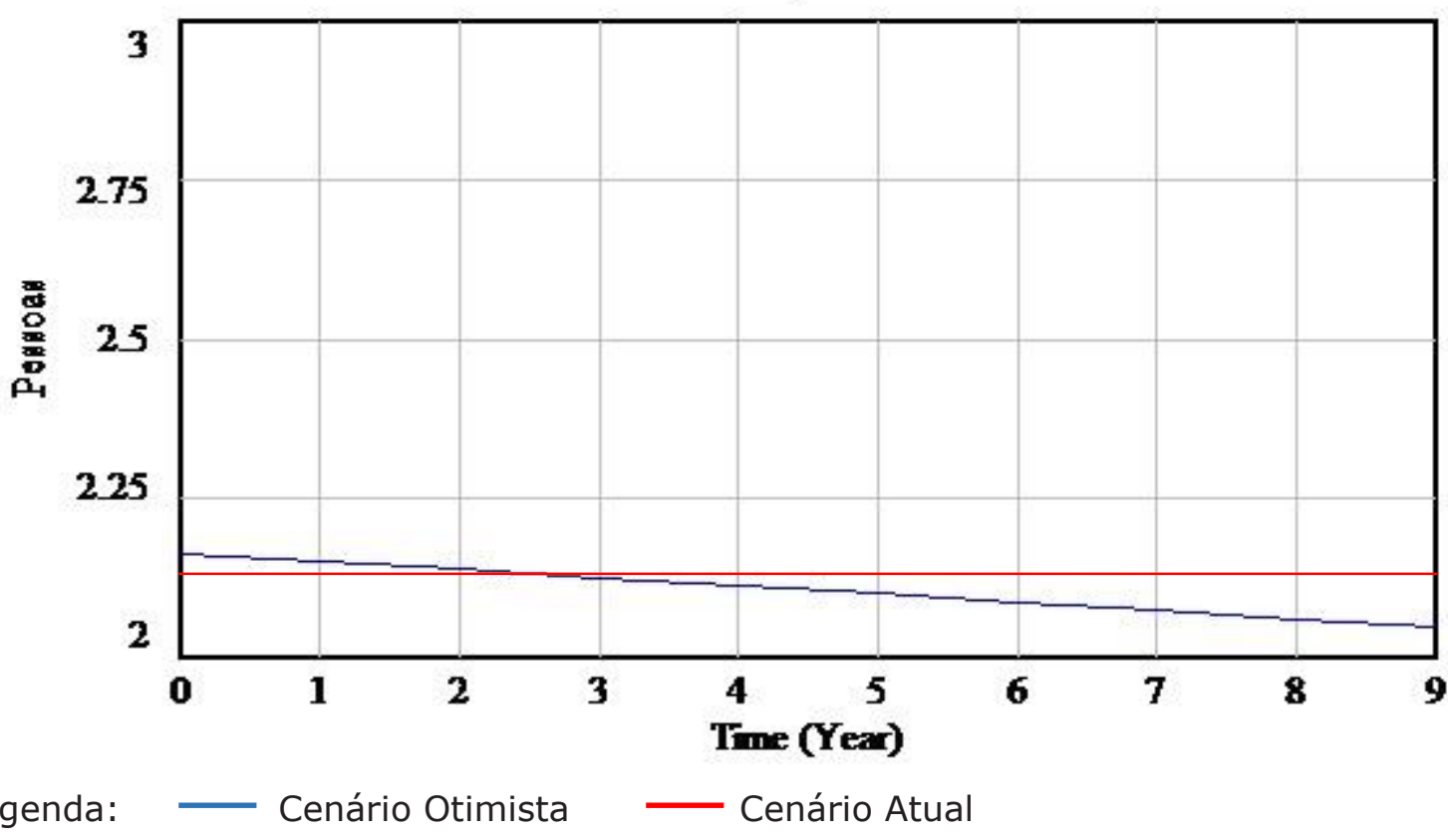

\section{Gráfico 2: Níveis de estresse ocupacional}

Fonte: Dados da Pesquisa

Analisando as informações contidas no Gráfico 2 e na Tabela 4, identifica-se que, com a manipulação das variáveis, os níveis de estresse ocupacional tendem a cair, fruto do aumento dos níveis de satisfação no trabalho, que tem relação inversamente proporcional com o estresse. Ou 
seja, ao se aumentarem os níveis de satisfação, reduzem-se os níveis de estresse. Esse achado corrobora o evidenciado por Lelis (2013), que também identificou o nível de estresse como inversamente proporcional ao nível de satisfação no trabalho.

Diante dos resultados observados destaca-se que, no final do $10^{\circ}$ período, o estresse estará reduzido ao nível de 2,05, em uma escala de 1 a 5. Sem a manipulação das variáveis "satisfação no trabalho" e "satisfação com as promoções", a média manter-se-ia em 2,16. O que significa dizer que, ao se aumentarem em $50 \%$ os níveis de satisfação com as promoções e satisfação com o salário, mantendo inalteradas as demais variáveis, obtém-se um crescimento nas médias de satisfação no trabalho, acarretando uma diminuição de 5,09\% nas médias de estresse.

Como forma de aplicar os resultados encontrados na presente pesquisa à realidade estudada, sugere-se elaborar e implantar um plano de carreira, em conjunto com representantes da Associação dos Sargentos, Subtenentes e Tenentes da BM (ASSTBM), baseado em conhecimentos, habilidades e atitudes, visando à valorização profissional e, consequentemente, da imagem da profissão. Além disso, aconselha-se também elaborar, implantar e atualizar constantemente um plano salarial em consonância com o plano de carreira, considerando-se as peculiaridades da profissão e também programas de incentivo.

\section{CONSIDERAÇÕES FINAIS}

O principal objetivo deste estudo foi apresentar o desenvolvimento e a validação de um modelo de simulação computacional que permita aos gestores da área de gestão de pessoas avaliarem e analisarem cenários acerca da satisfação no trabalho e do estresse ocupacional. No desenvolvimento deste estudo, foi considerado um período de dez anos, durante os quais se buscou aumentar os níveis de satisfação com promoções e satisfação com salário, procurando avaliar os efeitos gerados no estresse ocupacional.

Aumentando em $50 \%$ ao longo de todo o período os níveis de satisfação com salários e satisfação com as promoções, identificou-se um 
aumento nos níveis gerais de satisfação no trabalho em $14,8 \%$ ao longo dos dez períodos. A partir das relações inversamente proporcionais entre estresse e satisfação, encontradas em estudos de Almeida (2015) e Lelis (2013), estabeleceu-se uma associação entre as duas variáveis, considerando um impacto positivo da satisfação para a redução dos níveis de estresse. Diante da análise dessas relações, percebeu-se que o aumento da satisfação em $14,8 \%$ ao longo dos dez períodos gerou uma redução nos níveis de estresse ocupacional. Assim, o estresse foi reduzido de uma média de 2,16 a um nível de 2,05.

Os resultados desta pesquisa proporcionam uma importante compreensão acerca das relações da satisfação no trabalho com estresse ocupacional e fornecem um modelo de simulação que testa os impactos das ações desenvolvidas pela área de recursos humanos para elevar os níveis de satisfação sobre os níveis de estresse ocupacional de seus colaboradores. Além disso, permitem uma melhor análise de prospecção dos resultados esperados por meio da implementação de práticas e políticas de recursos humanos.

O modelo apresentado pode ser reaplicado ou adaptado a outras realidades organizacionais. Neste caso, aplicou-se na perspectiva dos quartéis da Brigada Militar do Estado do Rio Grande do Sul. Este modelo também pode ser melhorado, ou adaptado a outras realidades, com a inclusão de outras variáveis, que representem práticas e políticas de recursos humanos, visando, assim, simular cenários com maior poder de amplitude e explicação acerca do estresse ocupacional, como exemplo da socialização organizacional e do treinamento e desenvolvimento de pessoas. 


\section{REFERÊNCIAS}

Almeida, D. M. (2015). Satisfação no trabalho e estresse ocupacional na perspectiva dos policiais militares do estado do Rio Grande do Sul. Dissertação de Mestrado, Universidade Federal de Santa Maria, Santa Maria, RS, Brasil.

Brasil. Ministério da Saúde do Brasil. Organização Pan-Americana da Saúde/Brasil. (2001). Doenças relacionadas ao trabalho. Manual de procedimentos para os serviços de saúde. (série A. Normas e manuais técnicos, 114). Brasília, DF: Ministério da Saúde.

Chanlat, J. F. (2005). Mitos e realidades sobre o estresse dos gerentes. In: E. Davel, \& M. C. O. L. Melo, Gerência em ação: singularidades e dilemas do trabalho gerencial (pp. 29-65). Rio de Janeiro: FGV.

Costa, M., Accioly Jr., H., Oliveira, J., \& Maia, E. (2007). Estresse: diagnóstico dos policiais militares em uma cidade brasileira. Revista Panamericana de Salud Pública, 21(4), 217-22.

Daellenbach, H. G., \& McNickle, D. C. (2005). Decision making through systems thinking. London: Palgrave Macmillan.

Law, A. M., \& Kelton, W. D. (1991). Simulatin modeling \& analysis. New York: McGraw-Hill.

Lelis, J. W. F. (2013). Estresse e satisfação no trabalho de profissionais que exercem a função de gestores na área comercial. Dissertação de Mestrado, Pontifícia Universidade Católica de São Paulo, São Paulo: SP, Brasil.

Paschoal, T.; Tamayo, A. (2004). Validação da escala de estresse no trabalho. Estudos de Psicologia, 9(1), 45-52.

Robbins, S. P., Judge, T. A., \& Sobral, F. (2010). Comportamento organizacional: teoria e prática no contexto brasileiro (14a ed.). São Paulo: Pearson Prentice Hall.

Siqueira, M. M. M. (2008). Satisfação no trabalho. In: Siqueira, M. M. M. (Org.), Medidas do comportamento organizacional: ferramentas de diagnóstico e de gestão (pp. 29-40). Porto Alegre: Artmed.

Vasconcelos, T. S. (2011). Programas de gerenciamento do estresse e qualidade de vida no trabalho na área de segurança pública. In A. M. Rossi, P. L. Perrewé, \& J. A. Meurs (Orgs.), Stress e qualidade de vida no trabalho: stress social - enfrentamento e prevenção. São Paulo: Atlas.

Vensim-Ventana Systems. (2012). Vensim Simulation software. Recuperado em 15 de junho, 2015, de http://www.vensim.com. 